\title{
Real-time Momentary Mood as a Predictor of College Students' Prescription Drug Misuse in Daily Life: Direct Links and the Moderating Role of Background Mental Health
}

\author{
Lauren M. Papp, Ph.D. \\ University of Wisconsin-Madison \\ Chrystyna D. Kouros, Ph.D. \\ Southern Methodist University \\ Hannah K. Witt, B.S. and John J. Curtin, Ph.D. \\ University of Wisconsin-Madison \\ Shari M. Blumenstock, Ph.D. \\ Indiana University \\ Alexandra Barringer, M.S. \\ University of Wisconsin-Madison

\section{Experimental and Clinical Psychopharmacology \\ In press, April 2021}

\begin{abstract}
Author Note
Lauren M. Papp, Department of Human Development and Family Studies, University of Wisconsin-Madison. Chrystyna D. Kouros, Department of Psychology, Southern Methodist University. Hannah K. Witt, Department of Human Development and Family Studies, University of Wisconsin-Madison. John J. Curtin, Department of Psychology, University of WisconsinMadison. Shari M. Blumenstock, Kinsey Institute, Indiana University. Alexandra Barringer, Department of Human Development and Family Studies, University of Wisconsin-Madison.

We have no known conflict of interest to disclose. Research reported in this publication was supported by the National Institute on Drug Abuse of the National Institutes of Health under Award Number R01DA042093. The content is solely the responsibility of the authors and does not necessarily represent the official views of the National Institutes of Health.

Correspondence concerning this article should be addressed to Lauren M. Papp, Department of Human Development and Family Studies, University of Wisconsin-Madison, 1300 Linden Drive, Madison, WI 53706. E-mail: papp@wisc.edu
\end{abstract}




\begin{abstract}
The present study addressed calls for research to identify real-time predictors of prescription drug misuse (Schepis et al., 2020) by testing young adults' momentary reports of their negative mood and positive mood as predictors of event-level misuse in daily life. We implemented a 28day ecological momentary assessment (EMA) procedure that collected individuals' mood and other contextual experiences in moments preceding prescription drug misuse. Consistent with models of problematic substance use as a means to reduce negativity (Khantzian, 1997), results from hierarchical generalized linear modeling indicated within-person links between higher than usual negative mood and greater likelihood of prescription misuse in daily life. Contrary to the hypothesis, misuse was also more likely when preceded by elevated positive mood. We found consistent support for the hypothesized between-person effects, with prescription misuse in daily life associated with higher average levels of negative mood, and lower average levels of positive mood, across the reporting period. We further predicted that individuals reporting greater levels of social anxiety, depression, and externalizing symptoms would evidence stronger links between their momentary negative mood and prescription misuse. Partial support for this moderation hypothesis was found, with the positive within-person link between negative mood and prescription misuse significantly stronger among individuals higher (vs. lower) on social anxiety and depression. Results provide support for intricate connections between young adults' momentary mood, mental health symptoms, and prescription drug misuse.
\end{abstract}

Keywords: ecological momentary assessment; mental health; mood; prescription drug misuse; young adults

\title{
Public Significance Statement
}

This study identified elevated mood states-both positive and negative-as real-time predictors of young-adult college students' prescription drug misuse in daily life. Averaging across the 
course of the study, however, misuse was related to lower average positive mood and higher average negative mood. Students with higher levels of internalizing mental health symptoms (depression and social anxiety) were more likely to engage in misuse following moments of their elevated negative mood, pointing to modifiable risk factors to prevent and reduce college-based prescription drug misuse. 


\section{Real-time Momentary Mood as a Predictor of College Students' Prescription Drug Misuse in Daily Life: Direct Links and the Moderating Role of Background Mental Health}

As a population, young adults (between the ages of 18 and 25) report the highest rates of recent prescription drug misuse - defined as a person taking a medication intended for someone else, or taking their own medication for a different reason or in an alternative dose than intended - and those attending college demonstrate particular risks associated with this behavior, such as access to diverted medications or engaging in concurrent alcohol use (Tapscott \& Schepis, 2013). Generally, health-related functioning in this period has unique and lasting impact on the quality of adult development (Brown et al., 2008), and prescription misuse has been identified as a particular risk factor for later substance use disorders (McCabe et al., 2019). Given the significance and implications of this substance behavior among young adults, understanding modifiable psychosocial predictors of prescription drug misuse remains a priority.

The broader links between problematic substance use (such as prescription misuse) and affective states and mental health have strong conceptual and empirical backing. Selfmedication theory (Khantzian, 1997) postulates that individuals use various forms of substances to reduce their pain or discomfort and as a means to regulate negative emotions-an assertion that has been confirmed in studies of different populations and various forms of psychoactive substances (Mackesy-Amiti et al., 2015; Smith et al., 2017). In support of these theorized associations, robust connections have been established between mental health and prescription drug misuse behaviors specifically in young adult college students using retrospective reports. In a large sample of over 25,000 students drawn from 40 U.S. campuses (Zullig \& Divin, 2012), approximately $13 \%$ of the sample had endorsed any prescription drug misuse behavior (i.e., use of any painkiller, stimulant, sedative, or anti-depressant not prescribed to them in the past 12 months), and these students who misused also reported greater odds of several depressive symptoms and/or suicidality indicators; results varied somewhat by medication class. Teter and colleagues (2010) conducted an online survey of students at a large U.S. university that focused 
on misuse of prescription stimulants and found similar associations, with more frequent occasions of stimulant misuse in the past month (3+ times vs. 1-2 times) positively related to depressed mood experienced over the same time period.

In an interview-collected national sample of U.S. female college students $(N=2000)$ (McCauley et al., 2011), past-year misuse of prescription drugs (i.e., tranquilizers, sedatives, stimulants or pain medications) was endorsed by $7.8 \%$ of the sample, and, when predictors were assessed separately, misuse was positively linked to post-traumatic stress disorder and lifetime major depressive diagnoses, as well as substance use and victimization experiences. In the combined model, lifetime depression and substance use remained significant predictors, underscoring the mental health-substance use connections in this population. Drawing from a web-based survey of students $(N=1,052)$ attending a large public university (Tam et al., 2020), $24 \%$ of the sample endorsed recent misuse (multiple medication classes collapsed across lifetime use and recent use), and the authors reported more consistent links between misuse and depression symptoms compared to social anxiety symptoms. However, apparent differences in the symptom scales' reporting timeframes (i.e., over the past 3 months for depression, currently for social anxiety) could have accounted for the differential findings.

Whereas prior work has focused on the connections between college students' substance use and internalizing problems (Walters et al., 2018), there is limited evidence of links between prescription misuse and externalizing symptoms. Of note, Harries et al. (2018) obtained online survey responses regarding predictors of prescription opioid misuse from over 3,000 students at a large, public university in the U.S. Misuse was relatively infrequent $(2.2 \%$ endorsed in the past year, 5.3\% endorsed lifetime), yet was significantly and positively associated with alcohol and gambling problems, symptoms of anxiety, depression, and several externalizing measures, including gambling problems, impulsivity, and risky sex behaviors. The authors describe their findings as pointing to broader mental health risk factors to consider in 
the study of prescription drug misuse behaviors, yet note the inability of their design to determine temporal effects.

Cole and Hussong (2020) recently conducted a more nuanced test of whether prescription stimulant misuse and mental health were associated, accounting for individuals' broader substance patterns. Using a large sample of college students, they found that stimulant misuse along with other hard drug use (vs. stimulant misuse only) was more strongly linked to college students' impulsive tendencies. In contrast, their results did not suggest an association between stimulant misuse and depression symptoms (Cole \& Hussong, 2020). Their findings point to broad mental health correlates of medication misuse and further encourage moderation tests to identify when characteristics of the situation or instance might interact with background mental health factors of the individual. We draw from their framework to examine whether links between a person's mood in the moment (i.e., negative mood fluctuations across the day) and engaging in prescription drug misuse is stronger when the individual has higher levels of background mental health symptoms along internalizing (e.g., depression, social anxiety) or externalizing (e.g., impulsivity) dimensions.

In sum, a growing body of research has demonstrated that college students reporting elevated depression or other mental health symptoms are more likely to engage in prescription drug misuse (Zullig \& Divin, 2012). To date, this evidence is overwhelmingly based on retrospective, questionnaire measures and designs that capture mental health and/or prescription behaviors across varied or non-specified timeframes, leaving unknown how mental health experiences are associated with prescription misuse behaviors on an event level. Ecological momentary assessments (EMA), designed to capture multiple assessments of participants' lived experiences as they are happening (Shiffman et al., 2008), can assess mood states, behavioral intentions, and behaviors close to the time an event is experienced. The approach is able to capture reports of the hypothesized motivating factors before the substance behavior has occurred and thus represents a start to improving the temporal precision of 
research findings in this area (e.g., Huhn et al., 2016; see Singh \& Björling, 2019). Further, EMA yields repeated assessments of individuals that offer the ability to test within- and betweenperson hypotheses, and therefore support comparisons between a participant's own moments of misuse to non-misuse (within-person) in addition to comparing those people who report more versus less frequent misuse (between-person).

\section{Study Overview}

The present study heeded calls for research to identify ecologically-valid contextual correlates of prescription drug misuse (Schepis et al., 2020) by testing real-time reports of young adults' mood as predictors of prescription drug misuse in daily life. We designed and implemented an ecological momentary assessment (EMA) procedure that collected individuals' experiences right before prescription drug misuse occurred. Research aims and primary hypotheses were based on clinical conceptualizations and findings (e.g., Lo et al., 2013; Mackesy-Amiti et al., 2015) that have emphasized people's tendency to use substances to reduce their negative emotions and symptoms. The first research aim was to test whether fluctuations in negative mood and positive mood are associated with prescription drug misuse. In line with evidence for the direct role of affective states in substance use (Wills et al., 1999), we predicted that individuals would be more likely to engage in prescription drug misuse in their moments of higher-than-average negative mood and lower-than-average positive mood (withinperson effects). We also predicted that reporting higher average levels of negative mood, and lower average levels of positive mood, across the reporting period would be linked to engaging in prescription drug misuse in daily life (between-person effects). The second research aim was to examine the moderating role of background mental health on the link between negative mood and prescription drug misuse in daily life. We expected that individuals reporting greater levels of social anxiety, depression, and externalizing symptoms would evidence stronger links between their momentary negative mood and prescription misuse.

\section{Method}




\section{Participants}

Between September 2017 and September 2019, students at a large, public university in the Midwestern U.S. were continuously enrolled into an ongoing NIDA-funded (Masked award number) longitudinal study on daily behaviors and health in college life. The current study is drawn from the first phase of the larger project. Participants were recruited via flyers and announcements (e.g., newspaper ads, emails to enrolled students) that stated, "We are particularly interested in how people use prescription medications." Prospective participants completed an online screening and a telephone call was scheduled to confirm eligibility. Inclusion criteria were being enrolled as a freshman or sophomore and being 18 to 21 years of age. Based on screening, participants were oversampled for recent prescription misuse. The screening measure assured confidential responses and described, "Sometimes people use prescription drugs in ways that a doctor did not direct them to. Please think back over the past 3 months and consider whether you have used the following types of medications in any way a doctor did not direct you to use them, including using it without a prescription of your own; using it in greater amounts, more often, or longer than you were told to take it; or using it in any way a doctor did not direct you to use it." This question was asked for 4 prescription medication classes with common examples listed, including pain relievers (e.g., OxyContin, Percocet, hydrocodone, codeine, morphine, fentanyl, Vicodin), tranquilizers (e.g., Xanax, Ativan, Valium, Klonopin), stimulants (e.g., Dexedrine and Adderall, Ritalin, Concerta), and sedatives or barbiturates (e.g., Ambien, Lunesta, Sonata). Response options were Yes/No and prospective participants could endorse multiple misuse classes. Of the 355 total participants enrolled, 300 $(84.5 \%)$ endorsed misuse at screening. Of these 300 , most $(73.3 \%)$ endorsed recent misuse of one medication class at screening; the remainder endorsed misusing two or more classes. The majority of participants $(77.3 \%)$ reported recent misuse of prescription stimulants; the next highest class was pain medications ( $22.3 \%$ of the sample), followed by prescription tranquilizers (19.3\%), and prescription sedatives or barbiturates (11.3\%). These results are in line with 
national survey data showing college students to have the highest prescription misuse rates for stimulants (Schepis et al., 2018).

In terms of racial and ethnic background, $83.2 \%$ self-identified as White and $8.1 \%$ as Asian; nearly 7\% reported Hispanic or Latino/a background. Approximate undergraduate demographics on the campus were $71 \%$ White, 6.5\% Asian, and 5.3\% Hispanic or Latino/a. Remaining participants (8.4\%) self-identified as American Indian/Alaska Native, Black or African American, Native Hawaiian or Pacific Islander, or reported multiple or other races (individual identities included $<3 \%$ of respondents); one participant $(0.3 \%)$ did not respond. The racial and ethnic distribution of the sample was thus fairly consistent with state demographics at the time of the study (with Asian students overrepresented compared to state residents) but less diverse compared to students enrolled in U.S. institutions of higher education overall (Chronicle, 2019).

\section{Procedures}

Prior to the study, university institutional review board approval (Masked protocol number) and a National Institutes of Health Certificate of Confidentiality were obtained. Participants attended 2 lab sessions that were scheduled an average of 35 days apart and were trained to complete reporting procedures in daily life between the sessions. During the first session, participants completed informed consent procedures and survey measures (including demographic information) and were trained to use an iPod Touch application designed specifically for the present research (see Masked Citation for details); they chose a private password to access the application and completed a sample report in the lab. Access to all other device features was restricted. The scheduled reporting period started the following day. Although the typical reporting period was scheduled for the 28 days following the first lab session, some participants continued reporting until they returned their device at the second lab session; reports obtained across all days were retained in the current analyses to maximize statistical power for hypotheses testing. During the second lab session, participants returned their devices and completed additional measures. A research assistant then unlocked the 
device, downloaded the EMA reports to a secure server, and reset the devices. Participants received their choice of electronic or check payments; compensation included $\$ 75$ for session 1 , \$84 for reporting in daily life (prorated for partial completion), \$55 for session 2, and a \$36 bonus incentive for maintaining compliance across the planned reporting period.

Consistent with published EMA protocols on addictions (Buckner et al., 2015; Cooney et al., 2007; Preston \& Epstein, 2011), both signal-contingent and event-based assessments were administered. Signal-contingent reporting involved responding to a device prompt sent within four time windows (8:00 a.m.-11:30 a.m., 11:30 a.m.-3:00 p.m., 3:00 p.m.-7:00 p.m., and 7:00 p.m.-11:00 p.m.); prompts were sent at randomly varying times within each window across days. Participants were instructed to respond as soon as possible, as appropriate. Participants were also trained to self-initiate a report any time they intended to take one of four medications in any way a doctor did not direct them to use it (i.e., event-based assessment; see Measures). There was no limit on the timing or number of event-based reports that could be completed. To reduce burden, a signal-contingent prompt was not sent within 2 hours after a self-initiated, event-based report had been completed. EMA report questions included intentions to misuse (see Measures) and focused on participants' current location and social context and potential triggers of prescription drug misuse (e.g., mood states, pain, stressors, other substance behaviors). Each report included 28 questions; up to 12 additional questions were conditionally displayed (e.g., if nicotine use was endorsed, participant was prompted to select the type used). Signal-contingent and event-based assessments were identical and thus were indistinguishable in the resulting data files. If misuse intention of one or more of the medication classes was endorsed in the EMA report, participants were then sent a brief follow-up report 15 minutes later (Thrul et al., 2014) to assess misuse behaviors that might have occurred since the completion of the associated report; participants were instructed to respond to the follow-up within 15 minutes. All time-stamps of report and follow-up completion were automatically recorded.

\section{Measures}




\section{Outcome: Prescription Drug Misuse Behavior}

Following Buckner and colleagues' (2015) EMA protocol for cannabis use in daily life, participants were first asked about intentions to misuse a prescription drug. The EMA report asked, "Are you about to take a medication listed here in any way a doctor did not direct you to use it? Remember, this can include using a medication without a prescription of your own; using it in greater amounts, more often, or longer than you were told to take it; or using it in any other way a doctor did not direct you to use it." Participants indicated Yes or No for 4 classes of prescription drugs (examples of each were provided): sedatives or sleeping pills, tranquilizers or anxiety medications, stimulants, and pain relievers. In the follow-up report, participants were asked, "Have you recently taken a medication listed here, in any way a doctor did not direct you to use it?" The same 4 medication classes and examples were presented and participants responded No (0) or Yes (1). If any prescription misuse was endorsed across the four classes, this was considered an instance of prescription misuse, the behavior outcome of interest. Misuse was scored 1 when the behavior was endorsed on the follow-up, and 0 when the behavior was not endorsed on the follow-up or not administered due to responses of No to the intention questions. In instances when the intention questions were left blank (occurred on 57 EMA reports; described under Analytic Plan), the misuse variable was left as missing data.

\section{Momentary Predictors: Negative Mood and Positive Mood}

Each EMA assessment presented a list of 7 items of words and phrases and prompted

participants to, "rate your feelings and emotions right now, that is, at the present moment," on a scale from 1 (very slightly or not at all) to 5 (extremely) based on the PANAS-X (Watson \& Clark, 1999). Negative mood was calculated by summing momentary responses to the following items reflecting negative emotions: Fearful (afraid, scared, frightened, etc.), Hostile (angry, irritable, scornful, etc.), Guilt (ashamed, blameworthy, angry at self, etc.), and Sad (blue, downhearted, alone, lonely). Positive mood was calculated in the same way on the basis of items for Jovial (happy, joyful, delighted, etc.), Self-assured (proud, strong, confident, etc.), and 
Attentive (alert, concentrating, determined). Reliabilities were calculated at the between-person and within-person momentary levels (Cranford et al., 2006) for negative mood $\left(R_{\text {between }}=0.68\right.$, $\left.R_{\text {within }}=0.64\right)$ and positive mood $\left(R_{\text {between }}=0.79, R_{\text {within }}=0.76\right)$. It is worth noting that reliability for negative mood fell below values commonly deemed as adequate (Schmitt, 1996).

\section{Moderators: Background Mental Health}

Mental health scales were completed during the first lab session. Social anxiety was assessed by the Inventory for Anxiety and Depressive Symptoms - social anxiety subscale (IDAS; Watson et al., 2007). Participants rated the degree to which they felt or experienced particular feelings, sensations, problems, and symptoms over the past two weeks on a scale of 1 (not at all) to 5 (extremely). A sample item includes, "I found it difficult to talk with people I did not know well." Responses to the 5 symptoms of social anxiety were summed (Cronbach's $\alpha=$ .85). According to published clinical cut-points (Stasik-O'Brien et al., 2019), 17.6\% of our sample reported mild levels of social anxiety, $8 \%$ reported moderate levels, and less than $1.0 \%$ reported severe levels. The majority of the sample $(73.7 \%)$ did not report clinically meaningful levels of social anxiety symptomatology.

Depressive symptoms experienced in the past two weeks were assessed using the brief Patient Health Questionnaire (PHQ-9; Kroenke et al., 2001). The instrument asks, "How often have you been bothered by..." and includes items such as feeling down or depressed, appetitive disturbances, and feelings of failure. Participants respond on a scale of 0 (not at all) to 3 (nearly every day). The assessment has captured a range of depression in previous college-based studies (Garlow et al., 2008). Based on published cutoffs (Kroenke et al., 2001), depression severity in the current sample encompassed none (2.4\%), minimal $(24.2 \%)$, mild $(38.7 \%)$, moderate $(19.9 \%)$, and severe $(14.8 \%)$ levels. Continuous scores were used in the current analyses (9 items; Cronbach's $\alpha=.86$ ).

Externalizing behaviors and tendencies were assessed by the brief version of the Externalizing Spectrum Inventory (ESI-BF; Patrick et al., 2013). Specifically, the general 
disinhibition scale was used to assess externalizing and impulsive behavior, excluding substance use, mental health diagnoses, and aggression (Patrick et al., 2013; Rodriguez-Seijas et al., 2017). An example item is, "Things are more fun if a little danger is involved." Participants evaluated how much each statement reflects themselves using a 4-point Likert-type scale (false, somewhat false, somewhat true, and true). Responses were summed and scored such that higher scale scores indicate greater deviancy (20 items; Cronbach's $\alpha=.85)$.

\section{Analytic Plan}

As preliminary analyses, we examined descriptive statistics and tested correlations between person-level study variables. For the main research aims, we used multilevel modeling to examine within-person associations between momentary mood and prescription misuse behavior in daily life, and to test background mental health as a moderator. Models were run as a three-level hierarchical generalized linear model (HGLM) with Bernoulli distribution to account for the nested data (i.e., moments nested within days, days nested within people) and the binary dependent variable. Models were run using HLM v.8 (Raudenbush et al., 2019). We followed centering guidelines for disentangling within-person from between-person associations (Bolger \& Laurenceau, 2013). A benefit of this approach is that HLM uses all available reports and draws from all respondents, providing more weight to those with more data and, therefore, more reliable estimates. Additionally, HGLM has been utilized to model repeated assessments of substance outcomes that occur infrequently (e.g., relapse, Scott et al., 2018). Mood ratings at Level 1 were person-centered to represent the extent to which one's momentary positive (or negative) mood was higher or lower than their average positive (or negative) mood across the reporting period. The Level 1 model also included a variable for the moment within day (e. g., report number 1-4) to account for any systematic effects of reporting across the day on medication misuse. Due to minimal variability of misuse behaviors within days, the random effect corresponding to this term was not included. Level 2 accounted for Day; no day-level covariates were included. Individuals' average positive and negative mood levels (i.e., averaged 
across EMA reports) were included as a covariate at Level 3 (grand-mean centered). Participant sex was also included as an intercept control variable. Analyses tested the extent to which within-person (Level 1) fluctuations in momentary mood ratings directly predicted prescription drug misuse. Analyses further tested for between-person associations (Level 3), or the extent to which individuals who experienced higher levels of negative mood and positive mood, on average, also reported more prescription drug misuse behavior across the reporting period. Odds ratios were converted to Cohen's $d$ values to facilitate interpretation of effect sizes (Chinn, 2000).

The EMA reports in Level 1 included minimal missing data (mood or misuse intention variables were missing on $<0.01 \%$ of responses); these were left as missing and handled by HLM. There was no missing data that affected the scoring of the Level 3 mental health measures. Thus, no statistical adjustments for missingness were required.

To test whether background mental health moderated the within-person link between negative mood and prescription drug misuse, the social anxiety, depression, and externalizing scales were grand-mean centered and entered as Level 3 cross-level moderators of the slopes for the Level 1 mood predictors. These were also included as Level 3 intercept controls, along with the covariates described above. Each background mental health moderator was tested separately. The analysis plan was preregistered at Open Science Framework (https://osf.io/kjavw/). Additional exploratory analyses tested how negative mood and positive mood relate to misuse of the different medication types.

\section{Results}

\section{Preliminary Results}

Two participants did not return for the second lab session or return their data collection devices, and EMA data from 1 participant was not retrievable due to a device malfunction. The sample thus consisted of 297 participants (69\% female) with an average age of 19.5 years (SD $=0.71)$. Slightly more than half $(56.6 \%)$ were freshmen. The momentary data file included 
23,651 reports completed by 297 participants $(M=79.63$ reports, $S D=26.03$, range $=11-130)$. Participants' average EMA completion rate (calculated as the number of completed signalcontingent or self-initiated, event-based EMA EMA reports divided by the expected number of reports based on scheduled reporting days) and follow-up response rate (calculated as the number of completed follow-ups divided by the number of follow-up prompts sent following misuse intention of one or more of the medication classes in the EMA report) were $69 \%$ and $76 \%$, respectively, reflecting their consistent engagement and compliance with the procedure (see Masked Citation). Prescription drug misuse in daily life was endorsed by approximately one-third $(n=105)$ of the sample. These participants reported an average of 3.09 instances ( $S D$ $=3.12 ;$ range $=1-17)$ of misuse. In all, EMA reporting captured 331 instances of prescription drug misuse, including 5 instances of misuse of sedatives or barbiturates, 49 instances of misuse of tranquilizers, 260 instances of misuse of stimulants, and 17 instances of misuse of pain relievers.

Descriptive statistics and correlations among person-level variables are shown in Table 1. Averaged across all moments and days, participants' reports of their negative mood and positive mood in daily life were inversely associated. Average negative mood was associated with greater occurrence of prescription drug misuse in daily life, whereas positive mood was linked with less misuse occurrence in daily life. Negative mood and positive mood in daily life were related to the mental health scales (i.e., social anxiety, depression, and externalizing symptoms) in expected directions. The mental health scales were positively related to each other and the magnitude of these correlations indicated inter-related yet distinct constructs. Male participants reported higher average levels of positive mood in daily life.

\section{Direct Results}

The direct associations between mood and prescription drug misuse are shown in Table 2. Results from the model testing real-time negative mood as a predictor of misuse indicated that, in moments when negative mood was higher than usual (within-person), participants were 
more likely to engage in prescription drug misuse in daily life. Higher average level of negative mood across the reporting period (between-person) was also associated with increased likelihood of misuse. Results from the model testing real-time positive mood as a predictor of misuse indicated similar within-person but opposite between-person effects. In moments when positive mood was higher than usual (within-person), participants were more likely to engage in prescription drug misuse in daily life. Higher average level of positive mood (between-person) was associated with decreased likelihood of misuse in daily life. When negative and positive mood predictors were included simultaneously in one model, both the within-person and the between-person findings found in the separate models remained (Table 2). Across all models tested, prescription drug misuse was more likely to occur among females as compared to males, and misuse occurrence was not associated with the momentary report number of the day (Table 2). Effect sizes for all reliable results were very small.

\section{Moderation Results}

Results from moderation models testing cross-level interactions of the within-person link between negative mood and prescription drug misuse by mental health scales are shown in Table 3; scales were tested in separate models. Results yielded reliable support $(p<.05)$ for two of three hypothesized moderators: social anxiety and depression but not externalizing symptoms. Simple slope analyses were conducted by plotting $+1 S D$ and $-1 S D$ from the mean on the mental health moderators. Results from these tests indicated that the positive withinperson link between negative mood and prescription drug misuse was stronger among individuals reporting higher levels of social anxiety symptoms $(b=0.10, S E=0.009, A O R=$ $1.10, t=10.25, p<.001)$ compared to those reporting lower levels of social anxiety symptoms $(b=0.06, S E=0.01, A O R=1.06, t=4.37, p<.001)$, and among individuals reporting higher levels of depression symptoms $(b=0.09, S E=0.009, A O R=1.09, t=9.18, p<.001)$ compared to those reporting lower levels of depression symptoms $(b=0.06, S E=0.01, A O R=1.06, t=$ $4.17, p<.001)$. Levels of externalizing symptoms did not reliably moderate the within-person 
association between negative mood and prescription drug misuse in daily life (see Table 3). Effect sizes for all reliable results from the moderation and simple slope tests were in the very small to small range.

\section{Exploratory Analyses by Drug Type}

Exploratory tests assessed the associations between mood and prescription drug misuse by medication type. Given the low frequency of prescription misuse by drug type, exploratory analyses were limited to reports of misuse of stimulants and tranquilizers in daily life. Following the direct tests conducted using 3-level HGLMs (described above), negative and positive mood predictors were included simultaneously in one model, and participant sex and momentary report number were retained as covariates. Results indicated that participants were more likely to engage in prescription stimulant misuse in daily life in moments when their realtime negative mood and positive mood were higher than usual (within-person). Higher average level of negative mood across the reporting period (between-person) was also associated with increased likelihood of stimulant misuse. Results for tranquilizers followed a similar pattern. Participants were more likely to engage in tranquilizer misuse in daily life in moments when realtime negative mood and positive mood were higher than usual (within-person). Higher average level of negative mood (between-person) also predicted tranquilizer misuse. Documented effect sizes were small in magnitude (see Supplemental Table 1 for complete results).

\section{Discussion}

The current study was among the first to examine real-time predictors of young adults' prescription drug misuse in daily life. Analyses incorporated real-time assessments of negative and positive mood as potential triggers of prescription misuse behavior, along with potential moderating background measures of mental health symptoms. The novel design supported a temporally precise statistical test of within-person associations that revealed positive links between both positive and negative mood and prescription drug misuse. That is, college students were more likely to engage in prescription drug misuse in moments when they reported 
experiencing more positive mood and more negative mood than was typical for them in daily life. The within-person effect for negative mood was consistent with the hypothesized direction, whereas the direction of the within-person positive mood result was opposite of the hypothesized direction. The between-person hypotheses were consistently supported in that participants engaging in prescription misuse in daily life reported both higher levels of negative mood and lower levels of positive mood across the reporting period.

Theory suggests that problematic substance use occurs as a means to reduce negative mood (Khantzian, 1997), which provides a simple and reasonable explanation for the documented within- and between-person links between negative mood and prescription misuse behaviors. Interestingly, the unexpected finding of elevated momentary positive mood predicting misuse does align with recent evidence from a different college-based sample: Schepis et al. (2020) collected participants' reported motives for prescription stimulant misuse soon after the occurrence of the behavior in daily life. They found that positive mood reliably increased in the hours following misuse occurrence, and thus interpreted positivity enhancement - or the motivation to increase positive feelings - as a key driver of students' prescription stimulant misuse in daily life (Schepis et al., 2020). The differential (within- versus between-person) findings that we documented underscore the need to track these moods and prescription drug misuse over time to clarify implications of the behavior. For instance, a study of a similar college-based population (Cook et al., 2020) found that young adults who drank to reduce negative mood (versus to enhance positive mood) experienced more alcohol-related consequences.

The current study utilized a prescription misuse behavior outcome that combined across indications of misuse of multiple classes, consistent with other studies in the field and to overcome the relatively low frequencies for some medications found in our sample. Indeed, Schepis and Hakes (2011) drew from a U.S. nationally representative study of adults (over 2 waves of data) and found that lifetime and past-year prescription misuse (combined across 
medication classes) served as risk factors for the onset and occurrence of multiple forms of mental health disorders. Of note, stimulant misuse was the highest class endorsed in our sample, consistent with U.S. nationally representative findings (Schepis et al., 2018). We conducted post-hoc exploratory tests to improve understanding of the associations between mood and prescription misuse by drug type. Findings indicated that in-the-moment negative mood and positive mood were uniquely predictive of misuse of stimulants and tranquilizers in daily life. Higher average levels of negative mood across the reporting period also were consistently linked to greater likelihood of both types of prescription misuse, whereas overall positive mood experienced by participants was not reliably linked to misuse of either prescription stimulants or tranquilizers. These medication classes were also the most commonly misused in our sample and thus are likely to be the drug types driving the overall findings. The current study's primary and exploratory findings contribute novel information to guide future research in the field.

The moderating hypothesis (that young adults with higher levels of background mental health symptoms would evidence stronger links between their momentary negative mood and prescription misuse in daily life) received some support. In particular, the social anxiety and depression (but not externalizing) scales emerged as reliable moderators such that people reporting higher (versus lower) levels of the symptoms were more likely to misuse prescription drugs in response to experiencing greater negative mood than usual in daily life. A speculative interpretation at this point is that individuals in late adolescence and early adulthood with greater externalizing tendencies have heightened risk for problematic alcohol or other drug use (e. g., Farmer et al., 2016), whereas internalizing factors may underpin the heightened risk for prescription misuse in response to elevated negative mood. The present results contribute to a growing recognition of the potentially intricate role of prescription drug misuse in the varied connections between college students' substance use and mental health domains, and 
encourage future research to test such associations in prospective designs that track these variables over time (Cole \& Hussong, 2020).

\section{Clinical Implications}

The harmful mental and physical effects of misuse and abuse of prescription medications among college-based populations have been established (Daniels-Witt et al., 2017; Gomes et al., 2011). Noting that the problematic use of opioid prescriptions can be especially harmful or fatal, Kenne et al. (2017) conducted a large online survey of undergraduate and graduate students to survey them about their lifetime and past year misuse of prescription pain medications, as well as their reasons for misuse. Although the motive to relieve physical pain was more common than seeking relief from emotional pain, when the emotional pain motive was endorsed, students indicated particular embarrassment about seeking treatment and fear over going to a hospital or doctor for their distress (Kenne et al., 2017). Notably, college health settings have been described as ideal locations for screening for prescription drug misuse and diversion behaviors among students broadly and among those with other possible risk factors (e.g., being in a health-related area of study, Weyandt et al., 2020). Considered in the context of prior work, the current findings reinforce the connection between mental health problems and prescription misuse (Lo et al., 2013) and could motivate health professionals who treat college students for mental health distress to inquire about the students' experiences with prescription medication practices. This recommendation may become even more pressing, given that college students are expected to face long-term mental health consequences stemming from the ongoing COVID-19 pandemic (Wang et al., 2020).

\section{Limitations and Future Directions}

Similar to other college-based studies, our risk-based sample included an overrepresentation of females and White participants. Our comparison-group participants (not included in the current analytic sample) were significantly more diverse (Masked Citation). Sumstine and colleagues (2018) explored racial and ethnic variation in mental health correlates 
of multiple forms of substance use among students sampled from two colleges in California. Focusing on problematic prescription behaviors, their results found that White students had the highest rates of prescription stimulant and pain medication misuse, although students from Asian, Latino, and "all other" backgrounds also reported misuse rates that were higher than the national average. Racial and ethnic differences in associations between mental health symptoms and prescription misuse were identified; for example, White and Latino (but not Asian) students who scored higher on inattention were more likely to report misuse of stimulant medications (Sumstine et al., 2018). Other recent work based on highly disadvantaged populations found that unmet mental health needs and suicidal feelings were associated with recent prescription drug misuse among a U.S.-based sample of homeless young adults (Barman-Adhikari et al., 2019). Clearly, continued efforts are needed to understand the interplay of mental health challenges and prescription misuse behaviors among more diverse populations.

Despite our efforts in the current study to recruit a sample of participants with elevated likelihood of prescription drug misuse, most participants did not report misuse during the EMA period and the focal behavior was relatively infrequent (endorsed less than once per week on average). We thoroughly considered the methodological trade-offs of implementing an EMA design with an app based on a study-owned (versus a participant's own) device (Masked Citation). Using a study-owned device strengthened the privacy and security assurances we could provide to our participants, but likely resulted in missed instances of prescription drug misuse. Given our sound methods and statistical modeling, the present study's results reflect a conservative approach. Replication in designs that capture more instances of misuse would bolster confidence in the findings. Future suggestions include administering EMA methods on participants' own devices and enrolling participants with greater risk for the behavior. Among samples recruited on the basis of more stringent misuse criterion, college students misused stimulant medications slightly more than 2 times per week on average (Schepis et al., 2020). 
Furthermore, the average EMA report completion rate in our sample (based on the expected number of reports per participant) was lower than the pooled compliance rate $(75.06 \%)$ found in a large meta-analysis of EMA studies of substance use (Jones et al., 2018). The current study reported observed effect sizes that ranged from very small to small, which is consistent with evidence that multiple contextual factors pertaining to the situation and the individual contribute to prescription misuse occurrence (Schepis et al., 2020). In addition, the momentary mood variables demonstrated psychometric properties that were not ideal, with negativity alphas falling below values commonly deemed as adequate (Schmitt, 1996) and demonstrating lower reliability than positivity. Methodological attention to strengthening the assessment of these indicators at momentary (vs. daily or broader) intervals is needed.

Despite these limitations, the current study identifies momentary mood and background mental health indicators associated (both directly and in moderation models) with increased risk for engaging in prescription medication misuse in daily life. Results offer implications for future research and applied efforts to reduce a hazardous substance behavior during the important young-adulthood period, and encourage longitudinal assessments to support the identification of time-ordered dynamics between prescription drug misuse and mental health domains over time. 


\section{References}

Barman-Adhikari, A., Hsu, H.-T., Brydon, D., Petering, R., Santa Maria, D., Narendorf, S., Shelton, J., Bender, K., \& Ferguson, K. (2019). Prevalence and correlates of nonmedical use of prescription drugs (NMUPD) among young adults experiencing homelessness in seven cities across the United States. Drug and Alcohol Dependence, 200, 153-160. https://doi.org/10.1016/j.drugalcdep.2019.03.015

Bolger, N., \& Laurenceau, J.-P. (2013). Intensive longitudinal methods: An introduction to diary and experience sampling research. Guilford Press.

Brown, S. A., McGue, M., Maggs, J. L., Schulenberg, J., Hingson, R., Swartzwelder, S., ...Murphy, S. (2008). A developmental perspective on alcohol and youths 16 to 20 years of age. Pediatrics, 121, S290-S310. https://doi.org/10.1542/peds.2007-2243D

Buckner, J. D., Zvolensky, M. J., Crosby, R. D., Wonderlich, S. A., Ecker, A. H., \& Richter, A. (2015). Antecedents and consequences of cannabis use among racially diverse cannabis users: An analysis from Ecological Momentary Assessment. Drug and Alcohol Dependence, 147, 20-25. https://doi.org/10.106/j.drugalcdep.2014.12.022

Chinn, S. (2000). A simple method for converting an odds ratio to effect size for use in metaanalysis. Statistics in Medicine, 19, 3127-3131. https://dx.doi.org/10.1002/10970258(20001130)19:22<3127::AID-SIM784>3.0.CO;2-M

Chronicle of Higher Education (2019). Almanac of higher education: 2019-20. https://chronicle.com/almanac

Cole, V. T., \& Hussong, A. M. (2020). Psychosocial functioning among college students who misuse stimulants versus other drugs. Addictive Behaviors, 105. Advance online publication. https://doi.org/10.1016/j.addbeh.2020.106290

Cook, M. A., Newins, A. R., Dvorak, R. D., \& Stevenson, B. L. (2020). What about this time? Within- and between-person associations between drinking motives and alcohol 
outcomes. Experimental and Clinical Psychopharmacology, 28(5), 567-575. https://doi.org/10.1037/pha0000332

Cooney, N. L., Litt, M. D., Cooney, J. L., Pilkey, D. T., Steinberg, H. R., \& Oncken, C. A. (2007). Alcohol and tobacco cessation in alcohol-dependent smokers: Analysis of real-time reports. Psychology of Addictive Behaviors, 21, 277-286. https://doi.org/10.1037/0893164X.21.3.277

Cranford, J. A., Shrout, P. E., lida, M., Rafaeli, E., Yip, T., \& Bolger, N. (2006). A procedure for evaluating sensitivity to within-person change: Can mood measures in diary studies detect change reliably? Personality and Social Psychology Bulletin, 32(7), 917-929. https://doi.org/10.1177/0146167206287721

Daniels-Witt, Q., Thompson, A., Glassman, T., Federman, S. \& Bott, K. (2017). The case for implementing the levels of prevention model: Opiate abuse on American college campuses. Journal of American College Health, 65(7), 518-524. https://doi.org/10.1080/07448481.2017.1341900

Garlow, S. J., Rosenberg, J., Moore, J. D., Haas, A. P., Koestner, B., Hendin, H., \& Nemeroff, C. B. (2008). Depression, desperation, and suicidal ideation in college students: Results from the American Foundation for Suicide Prevention College Screening Project at Emory University. Depression \& Anxiety, 25(6), 482-488. https://doi.org/10.1002/da.20321

Gomes, J., Song, T., Godwin, L., \& Toriello, P. J. (2011). Prescription stimulant abuse on university campuses. Journal of Human Behavior in the Social Environment, 21(7), 822833. https://doi.org/10.1080/10911359.2011.615680

Harries, M. D., Lust, K., Christenson, G. A., Redden, S. A., \& Grant, J. E. (2018). Prescription opioid medication misuse among university students. The American Journal on Addictions, 27, 618-624. https://doi.org/10.1111/ajad.12807 
Huhn, A. S., Harris, J., Cleveland, H. H., Lydon, D. M., Stankoski, D., Cleveland, M. J., Deneke, E., \& Bunce, S. C. (2016). Ecological momentary assessment of affect and craving in patients in treatment for prescription opioid dependence. Brain Research Bulletin, 123, 94-101. https://doi.org/10.1016/j.brainresbull.2016.01.012

Jones, A., Remmerswaal, D., Verveer, I., Robinson, E., Franken, I. H. A., Wen, C. K. F., \& Field, M. (2018). Compliance with ecological momentary assessment protocols in substance users: A meta-analysis. Addiction, 114, 609-619. https://doi.org/10.1111/add.14503

Kenne, D. R., Hamilton, K., Birmingham, L., Oglesby, W. H., Fischbein, R. L., \& Delahanty, D. L. (2017). Perceptions of harm and reasons for misuse of prescription opioid drugs and reasons for not seeking treatment for physical or emotional pain among a sample of college students. Substance Use \& Misuse, 52(1), 92-99.

https://doi.org/10.1080/10826084.2016.1222619

Khantzian, E. J. (1997). The self-medication hypothesis of substance use disorders: A reconsideration of recent applications. Harvard Review of Psychiatry, 4, 231-244. https://doi.org/10.3109/10673229709030550

Kroenke, K., Spitzer, R. L., \& Williams, J. B. W. (2001). The PHQ-9: Validity of a brief depression severity measure. Journal of General Internal Medicine, 16(9), 606-613. https://doi.org/10.1046/j.1525-1497.2001.016009606.x

Lo, C. C., Monge, A. N., Howell, R. J., \& Cheng, T. C. (2013). The role of mental illness in alcohol abuse and prescription drug misuse: Gender-specific analysis of college students. Journal of Psychoactive Drugs, 45(1), 39-47. https://doi.org/10.1080/02791072.2013.763561

Mackesy-Amiti, M. E., Donenberg, G. R., \& Ouellet, L. J. (2015). Prescription opioid misuse and mental health among young injection drug users. The American Journal of Drug and Alcohol Abuse, 41(1), 100-106. https://doi.org/10.3109/00952990.2014.940424 
McCabe, S. E., Veliz, P. T., Boyd, C. J., Schepis, T. S., McCabe, V. V., \& Schulenberg, J. E. (2019). A prospective study of nonmedical use of prescription opioids during adolescence and subsequent substance use disorder symptoms in early midlife. Drug and Alcohol Dependence, 194, 377-385. https://doi.org/10.1016/j.drugalcdep.2018.10.027

McCauley, J. L., Amstadter, A. B., Macdonald, A., Danielson, C. K., Ruggerio, K. J., Resnick, H. S., \& Kilpatrick, D. G. (2011). Non-medical use of prescription drugs in a national sample of college women. Addictive Behaviors, 36, 690-695. https://doi.org/10.106/j.addbeh.2011.01.020

Patrick, C. J., Kramer, M. D., Krueger, R. F., \& Markon, K. E. (2013). Optimizing efficiency of psychopathology assessment through quantitative modeling: Development of a brief form of the Externalizing Spectrum Inventory. Psychological Assessment, 25(4), 13321348. https://doi.org/10.1037/a0034864

Preston, K. L., \& Epstein, D. H. (2011). Stress in the daily lives of cocaine and heroin users: Relationship to mood, craving, relapse triggers, and cocaine use. Psychopharmacology, 218, 29-37. https://doi.org/10.1007/s00213-011-2183-x

Raudenbush, S. W., Bryk, A. S., Cheong, Y. F., \& Congdon, R. (2019). HLM 8 for Windows [Computer software]. Scientific Software International, Inc.

Rodriguez-Seijas, C., Arfer, K. B., Thompson, Jr., R. G., Hasin, D. S., \& Eaton, N. R. (2017). Sex-related substance use and the externalizing spectrum. Drug and Alcohol Dependence, 174, 39-46. https://doi.org/10.1016/j.drugalcdep.2017.01.008

Schepis, T. S., Buckner, J. D., Klare, D. L., Wade, L. R., \& Benedetto, N. (2020). Predicting college student prescription stimulant misuse: An analysis from ecological momentary assessment. Experimental and Clinical Psychopharmacology. Advance online publication. https://doi.org/10.1037/pha0000386 
Schepis, T. S., \& Hakes, J. K. (2011). Non-medical prescription use increases the risk for the onset and recurrence of psychopathology: Results from the National Epidemiological Survey on Alcohol and Related Conditions. Addiction, 106(12), 2146-2155. https://doi.org/10.1111/j.1360-0443.2011.03520.x

Schepis, T. S., Teter, C. J., \& McCabe, S. E. (2018). Prescription drug use, misuse and related substance use disorder symptoms vary by educational status and attainment in U.S. adolescents and young adults. Drug and Alcohol Dependence, 189, 172-177. https://doi.org/10.1016/j.drugalcdep.2018.08.017

Schmitt, N. (1996). Uses and abuses of coefficient alpha. Psychological Assessment, 8, 350353. https://doi.org/10.1037/1040-3590.8.4.350

Scott, C. K., Dennis, M. L., \& Gustafson, D. H. (2018). Using ecological momentary assessments to predict relapse after adult substance use treatment. Addictive Behaviors, 82, 72-78. https://doi.org/10.1016/j.addbeh.2018.02.025

Shiffman, S., Stone, A. A., \& Hufford, M. R. (2008). Ecological momentary assessment. Annual Review of Clinical Psychology, 4, 1-32.

https://doi.org/10.1146/annurev.clinpsy.3.022806.091415

Singh, N. B., \& Björling, E. A. (2019). A review of EMA assessment period reporting for mood variables in substance use research: Expanding existing EMA guidelines. Addictive Behaviors, 94, 133-146. https://doi.org/10.1016/j.addbeh.2019.01.033

Smith, L. L., Yan, F., Charles, M., Mohiuddin, K., Tyus, D., Adekeye, O., \& Holden, K. (2017). Exploring the link between substance use and mental health status: What can we learn from the self-medication theory? Journal of Health Care for the Poor and Underserved, 28, 113-131. https://doi.org/10.1353/hpu.2017.0056

Sumstine, S., Cruz, S., Schroeder, C., Takeda, S., \& Bavarian, N. (2018). Racial/ethnic variation in mental health correlates of substance use among college students. Journal of 
Ethnicity in Substance Abuse, 17(2), 94-107.

https://doi.org/10.1080/15332640.2017.1300554

Tam, C. C., Benotsch, E. G., \& Weinstein, T. L. (2020). Resilience and psychiatric symptoms as mediators between perceived stress and non-medical use of prescription drugs among college students. The American Journal of Drug and Alcohol Abuse, 46(1), 120-130. https://doi.org/10.1080/00952990.2019.1653315

Tapscott, B. E., \& Schepis, T. S. (2013). Nonmedical use of prescription medications in young adults. Adolescent Medicine, 24, 597-610.

Teter, C. J., Falone, A. E., Cranford, J. A., Boyd, C. J., \& McCabe, S. E. (2010). Nonmedical use of prescription stimulants and depressed mood among college students: Frequency and routes of administration. Journal of Substance Abuse Treatment, 38, 292-298. https://doi.org/10.1016/j.jsat.2010.01.005

Thrul, J., Bühler, A., \& Ferguson, S. G. (2014). Situational and mood factors associated with smoking in young adult light and heavy smokers. Drug and Alcohol Review, 33, 420-427. https://doi.org/10.1111/dar.12164

Walters, K. S., Bulmer, S. M., Troiano, P. F., Obiaka, U., \& Bonhomme, R. (2018). Substance use, anxiety, and depressive symptoms among college students. Journal of Child \& Adolescent Substance Abuse, 27(2), 103-111.

https://doi.org/10.1080/1067828X.2017.1420507

Wang, X., Hedge, S., Son, C., Keller, B., Smith, A., \& Sasangohar, F. (2020). Investigating mental health of US college students during the COVID-19 pandemic: Cross-sectional survey study. Journal of Medical Internet Research, e22817. https://doi.org/10.2196/22817

Watson, D., \& Clark, L. A. (1999). The PANAS-X: Manual for the Positive and Negative Affect Schedule-Expanded Form [Unpublished manuscript]. Department of Psychology, University of lowa. 
Watson, D., O’Hara, M. W., Simms, L. J., Kotov, R., Chmielewski, M., McDade-Montez, E. A., Gamez, W., \& Stuart, S. (2007). Development and validation of the Inventory of Depression and Anxiety Symptoms (IDAS). Psychological Assessment, 19(3), 253-268. https://doi.org/10.1037/1040-3590.19.3.253

Weyandt, L. L., Gudmundsdottir, B. G., Holding, E. Z., Marraccini, M. E., Keith, M., May, S. E., Shepard, E., Francis, A., Wilson, E. D., Channell, I., \& Sweeney, C. (2020). Prescription opioid misuse among university students: A systematic review. Journal of American College Health. Advance online publication.

https://doi.org/10.1080/07448481.2020.1786095

Wills, T. A., Sandy, J. M., Shinar, O., \& Yaeger, A. (1999). Contributions of positive and negative affect to adolescent substance use: Test of a bidimensional model in a longitudinal study. Psychology of Addictive Behaviors, 13(4), 327-338.

https://doi.org/10.1037/0893-164X.13.4.327

Zullig, K. J., \& Divin, A. L. (2012). The association between non-medical prescription drug use, depressive symptoms, and suicidality among college students. Addictive Behaviors, 37, 890-899. https://doi.org/j.addbeh.2012.02.008 
Table 1

Descriptive Statistics and Correlations for Person-level Study Variables

\begin{tabular}{|c|c|c|c|c|c|c|c|c|c|}
\hline & $M($ or $n, \%)$ & $S D$ & 1 & 2 & 3 & 4 & 5 & 6 & 7 \\
\hline 1. Male & $92,31 \%$ & & - & & & & & & \\
\hline 2. Person-mean negative mood & 5.68 & 1.62 & -.02 & - & & & & & \\
\hline 3. Person-mean positive mood & 7.96 & 2.06 & $.17^{* *}$ & $-.23^{* *}$ & - & & & & \\
\hline 4. Occurrence of prescription misuse & 0.01 & 0.03 & -.11 & $.20^{* *}$ & $-.12^{*}$ & - & & & \\
\hline 5. Social anxiety & 11.52 & 4.57 & -.11 & $.35^{* *}$ & $-.27^{* *}$ & .04 & - & & \\
\hline 6. Depression & 8.41 & 5.45 & -.09 & $.55^{\star *}$ & $-.31^{\star *}$ & $.20^{* *}$ & $.56^{\star *}$ & - & \\
\hline 7. Externalizing & 15.62 & 8.74 & .06 & $.34^{* *}$ & $-.22^{\star *}$ & $.17^{* *}$ & $.29 * *$ & $.51^{* *}$ & - \\
\hline
\end{tabular}

Note. $N=297$ participants. Occurrence of prescription drug misuse was calculated as the sum of prescription misuse instances $/ n$ momentary reports for each participant.

${ }^{*} p<.05 .{ }^{* *} p<.01$. 
Table 2

Real-time Negative Mood and Positive Mood Predicting Prescription Drug Misuse in Daily Life:

Results from Generalized Multilevel Models

\begin{tabular}{lccc}
\hline Predictors & AOR & $p$ value & $95 \% \mathrm{Cl}$ \\
\hline \multicolumn{1}{l}{ Model 1: Negative mood } & & \\
Intercept & 0.02 & $<.001$ & {$[0.02,0.03]$} \\
Male & 0.72 & $<.001$ & {$[0.62,0.84]$} \\
Momentary report indicator & 1.05 & .11 & {$[0.99,1.11]$} \\
WP negative mood & 1.09 & $<.001$ & {$[1.07,1.11]$} \\
BP negative mood & 1.17 & $<.001$ & {$[1.13,1.22]$} \\
\hline & Model 2: Positive mood & & \\
Intercept & 0.03 & $<.001$ & {$[0.02,0.03]$} \\
Male & 0.74 & $<.001$ & {$[0.64,0.85]$} \\
Momentary report indicator & 1.02 & .37 & {$[0.97,1.08]$} \\
WP positive mood & 1.06 & $<.001$ & {$[1.04,1.08]$} \\
BP positive mood & 0.94 & $<.001$ & {$[0.91,0.97]$} \\
\hline & 1.13 & $<.001$ & {$[1.06,1.08]$} \\
Intercept & 1.07 & .004 & {$[0.95,0.99]$} \\
Male & 0.97 & $<.001$ & {$[1.09,1.12]$} \\
Momentary report indicator & Model 3: Negative mood and positive mood & {$[0.038,0.042]$} \\
WP negative mood & 0.04 & $<.001$ & {$[0.73,0.86]$} \\
BP negative mood & 0.79 & .26 & {$[0.99,1.05]$} \\
WP positive mood & 1.02 & $<.10,1.16]$ \\
BP positive mood & 1.10 & $<01$ & \\
\hline Note. $N=297$ participants. & 1.001 & \\
\hline
\end{tabular}

Note. $N=297$ participants. Male coded as female $=0$ and male $=1$. AOR $=$ adjusted odds ratio. $\mathrm{WP}=$ within-person. $\mathrm{BP}=$ between-person. 
Table 3

Link Between Negative Mood and Prescription Drug Misuse in Daily Life: Cross-level Interactions by Mental Health Symptoms from Generalized Multilevel Models

\begin{tabular}{lccc}
\hline & AOR & $p$ value & $95 \% \mathrm{Cl}$ \\
\hline Predicting slope of momentary negative mood on prescription drug misuse \\
Intercept of WP slope & 1.08 & $<.001$ & {$[1.07,1.10]$} \\
Moderator: Social anxiety & 1.004 & .017 & {$[1.001,1.007]$} \\
\hline Predicting slope of momentary negative mood on prescription drug misuse \\
Intercept of WP slope & 1.07 & $<.001$ & {$[1.05,1.10]$} \\
Moderator: Depression & 1.003 & .021 & {$[1.000,1.005]$} \\
\hline Predicting slope of momentary negative mood on prescription drug misuse \\
Intercept of WP slope & 1.10 & $<.001$ & {$[1.08,1.12]$} \\
Moderator: Externalizing & 1.003 & .055 & {$[1.000,1.006]$} \\
\hline
\end{tabular}

Note. $N=297$ participants. Models retained the control variables (participant sex and momentary report indicator) and included the moderator as a covariate of the intercept term. $\mathrm{AOR}=$ adjusted odds ratio. WP = within-person. 
Supplemental Table 1

Real-time Negative Mood and Positive Mood Predicting Prescription Drug Misuse (by

Medication Class) in Daily Life: Results from Generalized Multilevel Models

\begin{tabular}{lccc}
\hline Predictors & AOR & $p$ value & $95 \% \mathrm{Cl}$ \\
\hline & $Y=$ prescription stimulant misuse & \\
Intercept & 0.04 & $<.001$ & {$[0.03,0.05]$} \\
Male & 0.79 & .16 & {$[0.56,1.10]$} \\
Momentary report indicator & 0.94 & .015 & {$[0.90,0.99]$} \\
WP negative mood & 1.06 & .003 & {$[1.02,1.10]$} \\
BP negative mood & 1.13 & .014 & {$[1.03,1.24]$} \\
WP positive mood & 1.10 & $<.001$ & {$[1.07,1.13]$} \\
BP positive mood & 0.99 & .83 & {$[0.92,1.07]$} \\
& $Y=$ prescription tranquilizer misuse & \\
Intercept & 0.03 & $<.001$ & {$[0.025,0.027]$} \\
Male & 0.76 & $<.001$ & {$[0.70,0.82]$} \\
Momentary report indicator & 1.16 & $<.001$ & {$[1.14,1.18]$} \\
WP negative mood & 1.10 & $<.001$ & {$[1.09,1.11]$} \\
BP negative mood & 1.12 & $<.001$ & {$[1.10,1.15]$} \\
WP positive mood & 1.05 & $<.001$ & {$[1.04,1.06]$} \\
BP positive mood & 0.99 & .19 & {$[0.97,1.01]$}
\end{tabular}

Note. $N=297$ participants. Male coded as female $=0$ and male $=1$. AOR $=$ adjusted odds ratio. $\mathrm{WP}=$ within-person. $\mathrm{BP}=$ between-person. 